MICE-NOTE-GEN-84

A. Blondel

D. Kaplan

K. Long

M. Zisman

\title{
A brief case for the MICE experiment
}

This is a brief prepared for the NSF management to explain the particle and accelerator physics motivations for MICE. 
July 22,2004

\section{MICE, the International Muon Ionization Cooling Experiment}

The MICE experiment is being proposed by an international collaboration of 140 particle and accelerator physicists from Continental Europe, Japan, UK, and the US. It is intended to develop, and understand in detail, ionization cooling and its associated techniques. It is a crucial program in terms of designing and constructing a Neutrino Factory.

The observation of neutrino oscillations has revealed that neutrinos have mass, and mix. The fact that these masses are 6-12 orders of magnitude smaller than those of the other fundamental fermions constitutes one of the striking mysteries of fundamental physics today. Together with the unification of strong and electroweak forces, it may owe its origin to new physical phenomena at the grand unification scale (the so-called "see-saw" mechanism). The precise study of the pattern of mixing angles and of the mass hierarchy of neutrinos will provide unique clues into this uncharted domain. Moreover, the three family mixing naturally leads to leptonic $C P$ and $T$ violation, a leading explanation for the observed baryon asymmetry of the universe.

A "Neutrino Factory" based on a muon storage ring has been recognized as the ultimate tool for studies of neutrino oscillations, including possibly the discovery and study of leptonic $C P$ violation. It is also the first step towards a $\mu^{+} \mu^{-}$collider. The unique value of the Neutrino Factory is to provide an intense, pure, and well-defined beam of high-energy electron neutrinos. The quality of flux control will allow determination with unprecedented precision of the mixing parameters, mass differences, and $C P$ asymmetries. For example, the sensitivity - and precision — on the yet-unknown mixing angle $\theta_{13}$ outclasses other proposed facilities by more than one order of magnitude. The available neutrino energies extend up to the muon beam energy (typically 20-50 GeV), thus allowing the study of both $v_{\mathrm{e}} \rightarrow v_{\mu}$ and $v_{\mathrm{e}} \rightarrow v_{\tau}$ oscillations, as well as the detailed understanding of the matter resonance around $12 \mathrm{GeV}$. These features will allow precise determination of neutrino mixing parameters without ambiguities, and will provide unique tests of unitarity (in a way similar to the ongoing program for heavy flavor physics). None of the other proposed neutrino oscillation experiments, or combination thereof, has been shown to be as powerful.

These physics arguments provide strong motivation for the development of the key technologies leading to a Neutrino Factory as an international project, and justify the international composition of the MICE team and the joint involvement of experimental physicists and accelerator physicists.

Following earlier ideas, the concept of a Neutrino Factory emerged in the workshop on Physics at the Front-End of a Muon Collider, held at Fermilab in November 1997. It took root in the development of muon colliders by the Muon Collaboration in the US. In 1999-2001 two studies were carried out in the US leading to the conclusion that indeed a Neutrino Factory could be built with the desired performance, but that the cost was high 
(around \$2B). R\&D was estimated to be necessary both to reduce the cost by a sizeable factor and to ascertain the performance and cost of the key technologies.

Ionization cooling of muons is one of these key technologies. It is necessary to reach the high intensity needed to perform the physics program, but has never been demonstrated in practice. The aims of the international Muon Ionization Cooling Experiment are as follows:

- To show that it is possible to design, engineer, and build a section of cooling channel capable of giving the desired performance for a Neutrino Factory;

- To place it in a muon beam and measure its performance in various modes of operation and beam conditions, thereby investigating the limits and practicality of cooling.

The MICE collaboration has designed an experiment in which a section of an ionization cooling channel (based on the cooling channel designed for the second US feasibility study) is exposed to a muon beam. The proposed cooling channel comprises liquidhydrogen absorbers, providing energy loss, and high-gradient radio-frequency (RF) cavities to re-accelerate the particles, all tightly contained in a magnetic channel. It reduces the beam transverse emittance by $>10 \%$ for muon momenta between 140 and 240 $\mathrm{MeV} / \mathrm{c}$. Spectrometers placed before and after the cooling section perform the measurements of beam transmission and emittance reduction with an absolute precision of $\pm 0.1 \%$. The experiment involves a number of new aspects, such as high-gradient operation of RF cavities in a strong magnetic field, and measurement of emittance with high precision.

A proposal was submitted to the Rutherford Appleton Laboratory (RAL) in January 2003 and received a strong recommendation by the scientific review committee. Approval by RAL management followed in October 2003. The experiment has secured funding in the UK at the level of $£ 12.5 \mathrm{M}$ ( $£ 7.5 \mathrm{M}$ from the Office of Science and Technology, $£ 2.5 \mathrm{M}$ from PPARC, and a similar amount from the host laboratory). It has also received promised in-kind contributions from around the world, in the form of used RF power equipment from LBNL, Los Alamos and CERN, as well as a beam solenoid from PSI. Putting this together, the experiment is presently funded at the level of more than $50 \%$. The largest "missing" contributions are those from the US and from Italy. An additional contribution from Japan is also being actively sought, beyond the already committed Japanese contribution to the liquid-hydrogen absorber system. The US contribution would allow its scientists to play the role in the experiment appropriate to their seminal contributions to the design. The US proposal, submitted to the NSF in October 2002, requested $\$ 23.9 \mathrm{M}$ over 5 years. Subsequent R\&D has demonstrated a likely savings of at least $\$ 3 \mathrm{M}$ compared with the original estimate. Given the mix of university and national laboratory groups, it would be natural to share the experiment costs roughly equally between NSF and DOE. 
The experiment is by now well organized, with a charter, an elected management, and an intense level of activity. It has efficiently surmounted the difficulties of operating as an international collaboration by holding regular video and phone conferences as well as collaboration meetings in the various regions involved. Development of components for the experiment is progressing well: the first liquid-hydrogen absorber is under test at Fermilab, a prototype 201-MHz cavity is being built under the supervision of LBNL, and prototypes for the tracker and some of the other detectors have been exposed to test beams. The safety aspects associated with the proximity of liquid-hydrogen absorbers to RF cavities and particle detectors have been successfully reviewed with the help of external experts. Much has already been learned in the design and prototyping process.

Assuming proper funding and support, ionization cooling of muons could be demonstrated by 2008. In the same time frame, it is expected that a revised design will allow considerable reduction in the cost of a Neutrino Factory (there are excellent indications from the APS Neutrino Physics Study that this is possible) and that R\&D experiments on the other key issues, such as the target experiment proposed at CERN and the study of FFAGs undertaken in Japan (the PRISM experiment), should be completed. It will then be possible, and timely, for the international physics community to decide whether to undertake a Neutrino Factory. To meet this schedule, the muon beam line must be installed during the next long ISIS shutdown (likely in early 2006). This means that beam line component engineering and fabrication must get under way in early 2005 . UK Office of Science and Technology funding for this cannot be released without a commitment from the US to provide its share of the experimental equipment.

The proposed MICE program is thus a timely and crucial step towards this exciting physics program, which will probe the processes that occurred shortly after the Big Bang and likely led to the matter-filled Universe we observe today. 